\title{
Clima Organizacional: Análise Fatorial Confirmatória de Modelos de Mensuração Concorrentes
}

\author{
Daniel Ioshiteru Kinpara ${ }^{1}$ \\ Embrapa \\ Jacob Arie Laros \\ Universidade de Brasilia
}

\begin{abstract}
RESUMO - O objetivo deste trabalho foi verificar qual modelo de mensuração para a Escala Clima Organizacional (ECO) ajusta-se melhor aos dados. Quatro modelos concorrentes foram comparados: 1) o modelo de sete fatores de Laros e PuentePalacios; 2) um modelo de cinco fatores derivado do modelo anterior; 3) um modelo de seis fatores baseado na teoria de campo vital de Lewin; e 4) um modelo de três fatores baseado na teoria de motivação de McClelland. Foram analisados os dados de 9.901 respondentes da ECO. Os resultados de análise fatorial confirmatória indicaram o modelo de três fatores como o melhor. Todos os modelos mostram ajuste suficiente aos dados. Sugere-se que a escolha do modelo baseie-se na finalidade de uso da escala.
\end{abstract}

Palavras-chave: clima organizacional, modelos de mensuração concorrentes, análise fatorial confirmatória

\section{Organizational Climate: Confirmatory Factor Analysis of Competing Measurement Models}

\begin{abstract}
This study investigated which measurement model for the Scale of Organizational Climate (SOC) provided the best fit to the data. Four competing measurement models were compared: 1) the seven factor model of Laros and PuentePalacios; 2) a five factor model derived from the previous one; 3) a six factor model based on Lewin's field theory; and 4) a three factor model based on McClelland's motivation theory. The data of 9,901 persons who responded the SOC were used. Confirmatory factor analysis indicated the three factor model as the best model. All four models showed acceptable fit to the data. We advise to base the choice between the four competing models on the intended use of the scale.
\end{abstract}

Keywords: organizational climate, competing measurement models, confirmatory factor analysis

Nas últimas décadas, as organizações têm mostrado um interesse crescente por escalas organizacionais para lhes auxiliar no diagnóstico de eventuais problemas em seus ambientes de trabalho. Por meio desses instrumentos, os gestores podem tomar decisões e desenhar intervenções para modificar comportamentos e melhorar o desempenho de seus colaboradores. A escala de clima organizacional (ECO) é um exemplo de escala organizacional utilizada para esse fim (Laros \& Puente-Palacios, 2004). Essa escala busca apurar as múltiplas dimensões do construto clima organizacional (CLO). Apesar da sua popularidade, o construto CLO carece de consenso dentro da literatura de Psicologia Organizacional. James e Jones (1974) e James et al. (2008) realizaram um esforço considerável de síntese desse construto. Mesmo assim, várias dúvidas permanecem sobre o mecanismo pelo qual a melhoria do CLO implica em melhoria no desempenho da organização. Ainda que a teoria em torno desse construto não esteja clara, o uso do conceito CLO tornou-se muito difundido como prática organizacional. Inúmeros levantamentos de CLO são realizados, utilizados na tomada de decisão e divulgados. Carr, Schmidt, Ford e DeShon (2003) buscaram

1 Endereço para correspondência: Embrapa Cerrados, Caixa Postal 08223, Planaltina - DF. CEP 73310-970.

E-mail: daniel.kinpara@embrapa.br em literatura científica trabalhos empíricos em CLO publicados entre 1990 e 2000. Conseguiram reunir 51 estudos.

A relação ente CLO e desempenho da organização é complexa. Existem efeitos moderadores atuando sobre CLO (Wood \& Bandura, 1989; Schneider, Salvaggio, \& Subirats, 2002), efeitos mediadores de CLO (Ancarani, Di Marco, \& Giammanco, 2011) e outras variáveis antecedentes de produtividade que não apenas CLO (Nakata, Veloso, Fischer, \& Dutra, 2009; Ostroff, 1993; Patterson, Warr, \& West, 2004). De qualquer forma, é inegável a relação prática entre CLO e o desempenho da organização. Isso justifica o interesse na construção de escalas que buscam medir CLO. Porém, qual será a melhor forma de medir o construto?

As formas de medir CLO estão intimamente relacionadas aos diferentes entendimentos desse construto. Puente-Palacios (2002) apresenta quatro abordagens para CLO: estrutural, perceptual, interativa e cultural.

Baseado nessas diferentes abordagens, quatro modelos concorrentes foram desenvolvidos para medir CLO: (1) o modelo de sete fatores de Laros e Puente-Palacios (2004); (2) um modelo de cinco fatores derivado do modelo anterior; (3) um modelo de seis fatores baseado na teoria de campo vital de Lewin; e (4) um modelo de três fatores baseado na teoria de motivação de McClelland (1955). O modelo (1) será o modelo base (MB). Os modelos de (2) a (4) serão os modelos alternativos. 
O objetivo do presente trabalho é verificar qual modelo de mensuração para a Escala Clima Organizacional (ECO) se ajusta melhor aos dados. Os modelos serão comparados por meio de análise fatorial confirmatória (AFC). A hipótese é de que a melhor medida de CLO é do modelo de mensuração com o maior número de fatores.

A principal implicação teórica deste estudo é a determinação da teoria que melhor explique o construto CLO. A implicação prática é a construção de instrumento composto por itens fidedignos e fatores representativos do CLO. Instrumentos afinados diminuem o tempo e o custo de coleta e aumenta a sua abrangência de aplicação dentro da organização.

\section{O construto}

Um bom ponto de partida para compreender o construto de CLO é como ele se diferencia de outros construtos. Schneider et al. (2002) fazem uma interessante categorização de clima psicológico, CLO e cultura organizacional. Enquanto cultura organizacional é uma análise em nível da organização, clima psicológico e CLO são análises em nível do indivíduo. Por sua vez, a diferença entre esses dois tipos de clima é o compartilhamento da percepção. CLO lida com a percepção compartilhada dos indivíduos. Logo, é uma construção social. Existem outros entendimentos sobre clima e cultura organizacional. Por exemplo, para Menezes, Sampaio, Gomes, Teixeira e Santos (2009), a diferença é em termos de temporalidade. A cultura seria muito mais permanente. De qualquer forma, ambos os trabalhos concordam que clima e cultura são construtos distintos.

O processo de compartilhar envolve algum grau de consenso dentro do grupo. Os diferentes níveis de consenso intragrupo da percepção de CLO foram denominados por Schneider et al. (2002) de "força do clima".

Além do grau de consenso, o processo de construção desse consenso é distinto. Chan (1998) apresenta três modelos conceituais de composição do consenso: 1) um aditivo; 2) um consensual por mudança de referente (referent-shift consensus); e 3 ) um modelo de dispersão. A diferença entre eles é como o escore de CLO é composto, se por uma simples soma de percepções, se pela análise da variância dos escores individuais, ou se por uma nova conceituação das percepções dos indivíduos. Esse último modelo conceitual de Chan traz um interessante elemento. O consenso pode não ser apenas em torno da percepção de cada indivíduo, mas em torno da percepção do grupo criada a partir da percepção de cada indivíduo.

O grupo é um importante elemento em percepção de CLO. Diferentes grupos podem ter diferentes percepções e compartilhamentos. Estudando uma instituição de ensino, Arabaci (2010) observou que existem diferenças de percepção de CLO entre os funcionários administrativos e os professores de uma mesma instituição.

O que proporciona essa diferença entregrupos e intragrupos está relacionado com o próprio ambiente da organização. Um ambiente composto pela infraestrutura (organização) e pelas percepções de seus colaboradores (indivíduos). $\mathrm{O}$ trabalho de Stone e Gershon (2006) realizado em Unidades de Tratamento Intensivo (UTI) de hospitais apresentou um elemento adicional desse ambiente. Dependendo da atividade fim da organização, os elementos de infraestrutura e de percepção são mais ou menos determinantes do CLO.

Há outros elementos secundários como sexo e idade que influenciam na percepção de CLO. Numa instituição de ensino, pessoas mais idosas e mulheres tiveram percepção mais positiva de CLO do que homens e jovens (Arabaci, 2010).

\section{A medida de CLO}

Sá, Guimarães e Azevedo-Rosal (1998) verificaram que as primeiras propostas de medida de CLO partiram de uma perspectiva do indivíduo como unidade de análise, confundindo-se muito com a ideia de satisfação no trabalho. Bhutto, Laghari e Butt (2012) encontraram relação entre CLO e satisfação no trabalho estudando instituições bancárias. De 14 fatores relacionados ao CLO estudado por eles, cinco estavam também relacionados significativamente com satisfação no trabalho. Os fatores eram estrutura organizacional, identidade, relações humanas, equidade e empoderamento.

Essa proximidade entre satisfação no trabalho e CLO advém da ideia de CLO como um "ambiente realizador", motivador. Nesse contexto, satisfação é um reflexo do CLO. O problema dessa afirmação é que a premissa de causalidade não está confirmada. Um exemplo de medição de CLO a partir dessa perspectiva motivacional está no trabalho de Souza (1982). Nele, ela utilizou a escala de Kolb. Souza (1982) relata que a escala foi sensível para medir CLO e a situação motivacional. Mas ela reporta que houve diferenças nas medidas em função da natureza da tarefa e da posição do indivíduo na escala hierárquica da organização, observações estas confirmadas por Stone e Gershon (2006) e por Arabaci (2010). Souza (1982) relata que a organização ativamente atrai e traz indivíduos que reforçam os padrões culturais dela, o que evidencia o problema de causalidade. Gomes (2002) observou o mesmo.

Para McClelland (1972), CLO é a ferramenta para se administrar a motivação dos indivíduos. A teoria de motivação de McClelland (1972) tem sido usada com frequência para medir CLO. Gomes (2002) mediu o CLO de uma empresa de telecomunicações por meio dessa teoria. O autor agrupou as sete categorias da escala de CLO desenvolvida por Kolb (1978) nas três categorias de McClelland (1955): a dimensão de poder de McClelland agrupou as dimensões de liderança e conformidade de Kolb; a de realização agrupou responsabilidade, padrões de desempenho e clareza; e a de afiliação agrupou recompensas e calor humano e apoio. Gomes (2002) concluiu que foi possível medir CLO a partir de fatores do ambiente interno da empresa, porém esse não foi o único determinante da situação da empresa. Em vista da interferência de outros fatores nas medidas de CLO, essas medidas evoluíram na direção da percepção do indivíduo sobre conteúdos relacionados à organização (ambiente).

Estudando organizações de pesquisa e desenvolvimento (P\&D), Sbragia (1983) notou que motivação e envolvimento são intrínsecos à atividade desenvolvida pelos indivíduos desse tipo de organização. Para essas instituições, não fazia sentido preocupar-se em motivar as pessoas, mas em dar condições mais favoráveis para a realização de suas tarefas. 
Assim, CLO devia ser visto como uma preocupação de melhoria na organização.

A influência do ambiente sobre o indivíduo já vem sendo estudada desde a década de 1930. Em 1939, Kurt Lewin publica o primeiro artigo sobre a teoria do campo vital (Lewin, 1939). Mas somente com o estudo de Lewin, Lippitt e White (1939), quando se percebeu a importância de elementos culturais nos ambientes sociais, surgem os termos clima social e atmosfera social. Esses estudos de Lewin e um experimento conduzido por Elton Mayo em uma fábrica da Western Electric entre 1927 e 1933 dão o impulso para o surgimento da Escola de Relações Humanas da Teoria Geral da Administração. Essa escola caracteriza-se pela ênfase na influência da gerência sobre o desempenho dos trabalhadores e em um sistema social que determina o resultado do indivíduo (Maximiano, 2000). Somente em 1958, Argyris (1958) cunhou o termo clima organizacional. Entretanto, até a década de 1980, o termo "clima" se referia a clima social, ao invés de clima organizacional (Schneider, 1985). No Brasil, o estudo de Sbragia (1983) identificou 20 dimensões de CLO: conformidade, estrutura, recompensas, cooperação, padrões, conflito, identidade, estado de tensão, ênfase na participação, proximidade da supervisão, consideração humana, autonomia presente, prestígio obtido, tolerância existente, clareza percebida, justiça predominante, condições de progresso, apoio logístico proporcionado, reconhecimento proporcionado e forma de controle. No exterior, surgiu um modelo de CLO mais sintético com sete fatores (Kolb, Rubin, \& McIntyre, 1986): responsabilidade, padrões, recompensas, conformismo, calor e apoio, clareza organizacional e liderança. A partir do estudo de Sbragia (1983) e de Kolb et al. (1986), Menezes et al. (2009) propuseram um modelo de seis fatores: segurança, estratégia, remuneração, relação com a comunidade, liderança e desenvolvimento profissional.

\section{Os modelos}

Sabendo que CLO é um construto complexo, é certo que exista mais de um modelo concorrente. Thompson (2004) aponta outro motivo para procurar por outros modelos. Ele sugere desenvolver modelos verdadeiramente concorrentes a fim de, de fato, testar a robustez do modelo em estudo. Não simplesmente comparar esse modelo com modelos extremos como o modelo com fatores independentes ou o modelo saturado (ajuste perfeito).

Outro motivo para se procurar por modelos concorrentes está no alerta de Patterson et al. (2004). Estes autores afirmam que os estudos devem levar em conta duas dimensões: 1) uma dimensão específica-geral; e 2) uma dimensão descritiva-avaliativa. Para o presente trabalho, isso significa que se podem desenvolver modelos de CLO específicos-avaliativos, isto é, modelos cujo fim é determinar as ações de mudança em pontos específicos dentro da organização que impliquem em melhor desempenho. Ou que se podem desenvolver modelos de CLO cuja dimensão geral-explicativa seja mais relevante, ou seja, modelos cujo fim é de compreensão do fenômeno. Estes são modelos normalmente mais complexos, exploratórios, teóricos. Logo, para cada finalidade existem diferentes modelos.
Uma vez definida a finalidade do modelo, é importante estabelecer uma teoria que suporte o modelo escolhido. A falta de uma teoria pode levar a modelos cujo ajuste matemático é bom, mas que são difíceis de justificar. A título de comparação, Schulte, Ostroff, Shmylyian e Kinicki (2009) chegaram a um modelo de mensuração de CLO com 94 itens distribuídos em 11 fatores estudando organizações bancárias. Contudo, Laros e Puente-Palacios (2004) desenvolveram um novo modelo para organizações bancárias brasileiras a partir de um instrumento de 70 itens distribuídos em sete fatores proposto pela Empresa Brasileira de Pesquisa Agropecuária - Embrapa. Por meio de análise paralela e validação cruzada, eles chegaram a um modelo de escala de clima organizacional (ECO) com 43 itens distribuídos em sete fatores. Ainda assim, ao se estudar dois fatores desse modelo (Suporte Material e Valorização do Trabalho), existem indícios para um modelo mais parcimonioso composto de cinco fatores.

Além de justificar a escolha de um modelo, a teoria permite desenvolver novos modelos alternativos. Por exemplo, Guion (1973) propõe duas linhas de pensamento em CLO, uma dentro da abordagem estrutural e outra dentro da abordagem perceptual. A primeira, o autor entende CLO como um atributo da organização. A segunda, CLO é um atributo dos membros da organização. Como um atributo da organização, a medida de CLO traduz-se na acurácia da percepção da estrutura da organização. Como um atributo dos membros, é a medida de satisfação no trabalho ou das atitudes dos membros da organização. Este último faz do CLO um construto parecido ao de clima psicológico, i.e., a identificação das necessidades dos indivíduos, a satisfação delas e a criação de um clima "realizador", motivador (Gomes, 2002). Por sua vez, Nakata et al. (2009) seguem a abordagem interativa, na qual se define CLO como a relação entre a organização e os seus membros.

Conjugando as abordagens propostas por Puente-Palacios (2002) e as concepções de construto de CLO apresentadas por Guion (1973) e Nakata et al. (2009), pode-se elaborar três modelos alternativos: 1) um baseado na perspectiva de CLO como uma dimensão do indivíduo; 2) um na perspectiva de CLO como um atributo da organização; e 3) um na perspectiva de CLO como a relação entre o indivíduo e a sua organização. Estes três modelos alternativos coincidem com uma categorização de CLO apresentada por Moran e Volkwein (1992)

A partir desses entendimentos de CLO, o presente estudo comparou quatro modelos concorrentes de CLO a partir dos dados levantados em uma instituição bancária brasileira. $\mathrm{O}$ primeiro modelo concorrente será representado pelo modelo proposto por Laros e Puente-Palacios (2004), o qual foi obtido a partir de ajuste matemático do modelo proposto por Sá et al. (1998). O modelo de Sá e autores foi desenvolvido a partir de abordagem fenomenológica, na qual CLO é resultado de uma estrutura perceptivo-cognitiva. O instrumento de Sá et al. (1998) tem 74 itens distribuídos em sete fatores: gerenciamento centrado em pessoas e produção; comprometimento com a empresa; oportunidade de desenvolvimento e ascensão; equidade salarial; alcance de resultados organizacionais; condições de trabalho; e autogestão de equipes de trabalho.

O segundo modelo concorrente será um modelo parcimonioso de cinco fatores obtido por ajuste matemático do 
primeiro modelo. O terceiro modelo concorrente será o modelo baseado na teoria de campo vital de Lewin (CLO como atributo da organização). O quarto modelo concorrente será o modelo baseado na teoria de motivação de McClelland (CLO como atributo do indivíduo). A comparação será realizada por meio da análise fatorial confirmatória e seus indicadores de ajuste do modelo.

A perspectiva de CLO como a relação entre o indivíduo e a sua organização não foi considerada. O trabalho de Gavin (1975) mostrou que a interação não foi significativa, pelo menos para a instituição bancária que ele estudou. Logo, não foi desenhado um modelo conceitual representando essa interação.

Cabe notar que os dados utilizados nesta pesquisa provêm de um questionário cuja teoria utilizada no seu desenho é distinta das teorias de Lewin e de McClelland. Logo, diferenças conceituais entre os itens utilizados e os fatores dos modelos alternativos são esperadas.

\section{Método}

A fonte de dados do presente trabalho foi o estudo de Laros e Puente-Palacios (2004). Nele, a Escala de Clima Organizacional (ECO) foi submetida à validação cruzada por meio de análise fatorial exploratória (AFE). O trabalho chegou a uma estrutura fatorial de sete fatores composta por 43 itens que explicam 63,4\% da variância observada. O tamanho da amostra original era de 61.349 respondentes. Uma subamostra de 9.901 respondentes escolhidos aleatoriamente foi utilizada no presente trabalho. Os itens ${ }^{2}$ foram medidos por meio de escala Likert de seis pontos. Apesar de se constituir em uma escala discreta, a interpretação dos resultados foi feita assumindo os resultados como uma escala intervalar.

Utilizou-se o software Amos 18.0 (Arbuckle, 2009) do pacote estatístico PASW, da IBM, para o desenho dos modelos e para a análise fatorial. Foram seguidas as recomendações de desenho e análise preconizadas por Byrne (2001) e Thompson (2004).

Os dados foram submetidos à verificação de pressupostos para a realização da análise fatorial. A multicolinearidade foi verificada por meio do Fator de Inflação da Variância (FIV). A homocedasticidade e a linearidade dos dados foram verificadas por análise dos gráficos de regressão dos resíduos padronizados (ZResid) versus os valores preditos padronizados da regressão (ZPred). Os dados atípicos multivariados foram inspecionados por meio de gráficos do tipo scatterplots obtidos a partir de três regressões parciais. Foram escolhidas aleatoriamente três variáveis dependentes a partir das 43 variáveis disponíveis. As variáveis independentes foram as demais variáveis remanescentes, respectivamente. A independência dos termos de erro foi verificada por meio do teste de Durbin-Watson. Finalmente, a normalidade foi verificada pelo teste de Mardia aplicado a cada um dos modelos fatoriais antes do uso dos índices de modificação. Mesmo que

2 Por acordo de confidencialidade com a organização onde os questionários foram aplicados, os itens do instrumento não puderam ser divulgados. o pressuposto de normalidade seja violado, não se observa problemas com as estimativas por máxima verossimilhança (maximum likelihood) para amostras maiores que $2.500(\mathrm{Hu}$ et al. citado por Tabachnick \& Fidell, 2001, p. 697).

Depois da verificação dos pressupostos, quatro modelos de mensuração concorrentes foram desenvolvidos no pacote Amos: 1) um modelo com sete fatores (gestão das relações interpessoais, comprometimento organizacional, trabalho em equipe, carga de trabalho, expectativa de desempenho, suporte material e valorização do trabalho), conforme obtido por Laros e Puente-Palacios (2004), denominado de Modelo Base (MB); 2) um modelo com cinco fatores (gestão das relações interpessoais, comprometimento organizacional, trabalho em equipe, carga de trabalho e expectativa de desempenho) obtido a partir de indícios de um modelo mais parcimonioso, denominado de Modelo Alternativo 1 (MA1); 3) um modelo com seis fatores (segurança, estratégia, remuneração, liderança, relação com a comunidade e desenvolvimento profissional) baseado na teoria de campo vital de Lewin, com foco na organização, conforme proposto por Menezes et al. (2009), denominado de Modelo Alternativo 2 (MA2); e 4) um modelo com três fatores (poder, realização e afiliação) baseado na teoria de motivação de McClelland (1955), denominado de Modelo Alternativo 3 (MA3).

A distribuição dos itens nos fatores dos modelos alternativos foi feita por meio de análises fatoriais exploratórias pelo método de fatoração dos eixos principais, com rotação oblíqua Promax, utilizando diferentes critérios de extração. Fixou-se o número de fatores a se extrair em seis (MA2), cinco (MA1) e três fatores (MA3). Suprimiram-se coeficientes menores que 0,32 . Os agrupamentos de itens obtidos foram utilizados para a descrição dos fatores de cada modelo. Nessa etapa exploratória, utilizaram-se todos os 9.901 respondentes.

Uma vez obtidos os fatores, construiu-se os modelos alternativos de maneira a que todos incluíssem um Fator Geral (FG) de segunda ordem. Contudo, não se pode afirmar que FG é o construto CLO em todos os modelos porque se utilizaram a teoria de campo vital e a teoria de motivação para MA2 e MA3, respectivamente.

Os modelos de mensuração elaborados foram submetidos à análise fatorial confirmatória (AFC) pelo método de estimação por máxima verossimilhança. Aqui se utilizaram os 9.901 respondentes. A partir dos índices de modificação, procedeu-se à alteração do modelo ao longo de várias iterações. As alterações foram realizadas até o momento em que os indicadores de ajuste não mostravam melhora acentuada.

Para a comparação do ajuste dos modelos, utilizaram-se três conjuntos de indicadores (Brown, 2006): 1) ajuste absoluto do modelo; 2) ajuste corrigido para parcimônia; e 3) ajuste comparativo. Para o indicador de ajuste absoluto, adotou-se o Standardized Root Mean Square Residual (SRMR) sugerido por Brown (2006). A partir da sugestão de Tabachnick e Fidell (2001), o indicador parcimonioso escolhido foi o Akaike Information Criterion (AIC) de Akaike (1987), pois os modelos estudados não são aninhados (non nested). Para o indicador comparativo, foram escolhidos dois cálculos: o Root Mean Square Error of Approximation (RMSEA) e o Comparative Fit Index (CFI). O RMSEA compara o modelo obtido com o modelo saturado, enquanto o CFI compara com uma distribuição $\chi^{2}$ não-central. 
Tabela 1. Indicadores de ajuste dos modelos (número de fatores), depois (antes) da análise dos índices de modificação, amostra de 9.901 respondentes.

\begin{tabular}{llllll}
\hline Modelo & SRMR & AIC & RMSEA & CFI & Itens \\
\hline MB (7) & $0,057(0,059)$ & $14.839(24.740)$ & $0,052(0,053)$ & $0,918(0,905)$ & $34(43)$ \\
MA1 (5) & $0,050(0,061)$ & $11.493(32.275)$ & $0,053(0,062)$ & $0,927(0,871)$ & $30(42)$ \\
MA2 (6) & $0,056(0,059)$ & $15.202(28.358)$ & $0,051(0,058)$ & $0,920(0,888)$ & $35(42)$ \\
MA3 (3) & $0,049(0,066)$ & $6.550(33.832)$ & $0,055(0,078)$ & $0,948(0,846)$ & $22(35)$ \\
\hline
\end{tabular}

Nota. SRMR = Standardized Root Mean Square Residual; AIC = Akaike Information Criterion; RMSEA = Root Mean Square Error of Approximation; CFI = Comparative Fit Index. MB = Modelo Base; MA1 = Modelo Alternativo 1; MA2 = Modelo Alternativo 2; MA3 = Modelo Alternativo 3.

Foram realizados dois conjuntos de teste de significância: um teste denominado de vertical, comparando o modelo original e o seu par modificado; e um teste denominado de horizontal, comparando os quatro modelos entre si. Para o teste vertical, os resultados de $\chi^{2}$ dos modelos foram submetidos a teste de significância conforme sugerido por Thompson (2004). Para o teste horizontal, por se tratarem de modelos não-aninhados, seguiu-se sugestão de Hélie (2006). Utilizaram-se as técnicas de bootstrapping e de cross-validation. Para o bootstrapping, realizaram-se 200 amostragens aleatórias com reposição para cada modelo. A comparação entre modelos foi feita a partir das estimativas de $\chi^{2}$ obtidas pelo bootstrapping e seus respectivos intervalos de confiança. Para o cross-validation, a amostra total foi dividida em 10 subamostras de 990 observações obtidas aleatoriamente e sem reposição. Dez "rounds" de AFC foram executados. Cada round era composto por uma "subamostra-teste" e uma "subamostra-treino". Em cada round, uma subamostra diferente era escolhida como subamostra-teste. As nove subamostras restantes eram combinadas em uma única subamostra-treino. $\mathrm{O}$ resultado dos 10 rounds geraram 10 valores de Expected Cross-Validation Index (ECVI) e seus respectivos intervalos de confiança. Calculou-se a média dos 10 valores. Esta média foi utilizada como representativa do modelo e meio de comparação entre modelos.

Os fatores assim obtidos foram submetidos à análise de fidedignidade pelo coeficiente Lambda-2 de Guttman ( $\lambda 2$ ). Nenhuma das escalas era reversa, não sendo necessário proceder à sua inversão.

\section{Resultados}

O resultado da média do FIV foi 2,33, abaixo do critério de 10 de Myers (1990), o que indica pouco problema de multicolinearidade. A inspeção visual dos gráficos de resíduos não apontaram problemas acentuados de linearidade ou homocedasticidade. O teste de Durbin-Watson resultou em valores de 1,89 a 2,06. Valores em torno de 2,00 significam que os resíduos adjacentes não estão correlacionados. Os resultados normalizados de curtose multivariada de Mardia foram 1,25, 1,19, 1,25 e 1,14 para os modelos de sete, cinco, seis e três fatores, respectivamente. Esses valores não indicam problemas de normalidade por apresentarem valores inferiores a 3,00 (Ullman, 2006). Em função desses resultados, nenhuma transformação dos dados foi realizada.

A Tabela 1 apresenta os resultados das análises fatoriais confirmatórias (AFC) dos modelos propostos. Note que os resultados se referem a antes e depois de alteração pelos índices de modificação.

Na Tabela 2 estão os resultados do teste vertical de significância, entre o modelo original (sem modificação) e o modelo modificado. Como os testes indicaram diferença significativa entre os modelos original e modificado a $p<0,001$, o teste de significância horizontal foi realizado apenas entre os modelos modificados, uma vez que estes apresentaram desempenho melhor que os modelos não modificados.

Na Tabela 3 estão as médias de ECVI, com os respectivos intervalos de confiança a $95 \%$, dos quatro modelos concorrentes obtidos pelas técnicas de bootstrapping e cross-validation. Note que as comparações de ECVI foram feitas entre as estimativas do modelo (implied) e os dados

Tabela 2. Teste de significância entre os modelos (número de fatores) original e modificado, amostra de 9.901 respondentes.

\begin{tabular}{|c|c|c|c|c|c|c|}
\hline Modelo & Modificação & $\chi^{2}$ & GL & $\Delta \chi^{2}$ & $\Delta \mathbf{G L}$ & $p$ calculado \\
\hline \multirow{2}{*}{ MB (7) } & Não & 24.554 & 853 & \multirow{2}{*}{9.865} & \multirow{2}{*}{333} & \multirow{2}{*}{$<0,001$} \\
\hline & Sim & 14.689 & 520 & & & \\
\hline \multirow{2}{*}{ MA1 (5) } & Não & 32.097 & 814 & \multirow{2}{*}{20.734} & \multirow{2}{*}{414} & \multirow{2}{*}{$<0,001$} \\
\hline & Sim & 11.363 & 400 & & & \\
\hline \multirow{2}{*}{ MA2 (6) } & Não & 28.178 & 813 & \multirow{2}{*}{13.128} & \multirow{2}{*}{259} & \multirow{2}{*}{$<0,001$} \\
\hline & Sim & 15.050 & 554 & & & \\
\hline \multirow{2}{*}{ MA3 (3) } & Não & 33.686 & 557 & \multirow{2}{*}{27.230} & \multirow{2}{*}{351} & \multirow{2}{*}{$<0,001$} \\
\hline & Sim & 6.456 & 206 & & & \\
\hline
\end{tabular}

Nota. $\mathrm{MB}=$ Modelo Base; MA1 = Modelo Alternativo 1; MA2 = Modelo Alternativo 2; MA3 = Modelo Alternativo 3. 
Tabela 3. Média da estimativa da Expected Cross-Validation Index (ECVI), média do erro padrão da média (EP) e cálculo do Intervalo de Confiança (IC) a $95 \%$ obtidos por meio de bootstrapping (cross-validation) dos modelos modificados (número de fatores), com valores estimados comparados com a amostra.

\begin{tabular}{|c|c|c|c|c|}
\hline Modelo & Média ECVI & Média EP & IC Inferior & IC Superior \\
\hline MB (7) & $15.338(1,581)$ & $23(0,024)$ & $15.293(1,533)$ & $15.383(1,628)$ \\
\hline MA1 (5) & $11.893(1,223)$ & $20(0,021)$ & $11.854(1,182)$ & $11.932(1,265)$ \\
\hline MA2 (6) & $15.757(1,620)$ & $23(0,025)$ & $15.712(1,571)$ & $15.802(1,669)$ \\
\hline MA3 (3) & $6.716(0,696)$ & $15(0,016)$ & $6.687(0,665)$ & $6.745(0,728)$ \\
\hline
\end{tabular}

Nota. A cross-validation foi realizada por meio de dez subamostras de 990 respondentes. MB = Modelo Base; MA1 = Modelo Alternativo 1; MA2 = Modelo Alternativo 2; MA3 = Modelo Alternativo 3.

da amostra (sample). Verificam-se diferenças entre os resultados do bootstrapping e do cross-validation. Enquanto no bootstrapping todos os modelos são significativamente diferentes, no cross-validation, MA2 e MB não se diferenciam significativamente.

Na Tabela 4 estão os resultados do $\lambda 2$ de Guttman, bem como o número de itens presentes em cada fator. Note que os fatores apresentados na Tabela 4 não guardam semelhanças entre si. Por exemplo, o fator F1 do primeiro modelo não representa a mesma variável latente do fator F1 dos demais modelos.

A diferença entre os fatores fica mais clara observando a Tabela 5. Os itens também mostraram correlação item-total corrigida sempre maior do que 0,32 . Os resultados de porcentagem da variância explicada pelos modelos modificados foram: $63,4 \%, 58,0 \%, 60,9 \%$ e 49,8\%, para MB, MA1, MA2 e MA3, respectivamente.

\section{Discussão}

Comparando-se os valores da Tabela 1 , fica evidente a sensível melhoria dos indicadores de ajuste após análise e alteração dos modelos por meio dos índices de modificação. Nota-se que o grau dessa melhoria coincidiu, de certa forma, com o número de fatores do modelo. Quanto maior o número, menor o efeito dos índices de modificação. Isso é um pouco contraintuitivo, uma vez que se espera que quanto mais fatores tenha o modelo, maior a possibilidade de modificações. Outra explicação pode estar na fonte de dados. Como o questionário aplicado foi desenvolvido utilizando o modelo com mais fatores (MB), a diferença entre o modelo obtido e os dados ficaria menor, o que resultaria numa menor necessidade de modificação do modelo. Seja qual for a explicação para essa diferença de efeito dos índices de modificação, está claro que quanto mais fatores tem o modelo, menos modificações são necessárias. Logo, neste estudo, os modelos com mais fatores podem não apresentar o melhor ajuste aos dados, mas são modelos estruturalmente mais robustos, i.e., pouco se modificam.

Os resultados apresentados na Tabela 2 indicam que a diferença entre o modelo sem modificação e com modificação é significativa. Como os resultados dos ajustes foram melhores com o modelo modificado, as demais análises dos resultados serão feitas sobre esse modelo.

Por meio da Tabela 1, não é possível comparar diretamente os diferentes modelos entre si, pois não são modelos aninhados. Ainda assim, é pertinente comparar os diferentes indicadores de um mesmo modelo. Observando esses valores, duas afirmações podem ser feitas: 1) os indicadores não foram convergentes entre si; 2) esse padrão não convergente foi igual em todos os modelos concorrentes. Enquanto SRMR e RMSEA consideram o modelo em análise com um bom ajuste (SRMR $<0,08$ e RMSEA $<0,06$ ), CFI indica um ajuste abaixo de "bom" (CFI $<0,95)$, porém suficiente $(\mathrm{CFI}>0,90)$. Essas diferenças podem ser explicadas pelo fato desses indicadores medirem aspectos diferentes do ajuste. Enquanto SRMR e RMSEA calculam os ajustes com base em resíduos, o CFI calcula o ajuste com base na comparação entre o modelo obtido e o modelo independente. Assim, SRMR e RMSEA comparam diferenças de ajustes e o CFI compara desempenho do ajuste. Logo, os modelos apresentam-se bons na medida do construto (pouco resíduo), mas com um desempenho de ajuste não tão melhor do que o modelo independente.

Tabela 4. Valores de $\lambda 2$ de Guttman (número de itens) de cada fator de Modelo Base (MB), Modelo Alternativo 1 (MA1), Modelo Alternativo 2 (MA2) e Modelo Alternativo 3 (MA3), após ajuste pelos índices de modificação.

\begin{tabular}{|c|c|c|c|c|c|c|c|c|}
\hline Modelo & F1 & F2 & F3 & F4 & F5 & F6 & F7 & $\begin{array}{c}\text { Total de } \\
\text { Itens }\end{array}$ \\
\hline MB & $0,92(8)$ & $0,88(7)$ & $0,82(6)$ & $0,72(2)$ & $0,81(4)$ & 0,74 (4) & 0,87 (3) & 34 \\
\hline MA1 & 0,93 (9) & $0,88(7)$ & $0,82(6)$ & $0,79(6)$ & $0,76(2)$ & --- & --- & 30 \\
\hline MA2 & 0,74 (4) & $0,84(6)$ & $0,85(3)$ & 0,93 (9) & $0,84(6)$ & $0,88(7)$ & --- & 35 \\
\hline MA3 & 0,93 (12) & $0,88(7)$ & $0,85(3)$ & --- & --- & --- & --- & 22 \\
\hline
\end{tabular}


Tabela 5. Variáveis latentes representadas pelos fatores de Modelo Base (MB), Modelo Alternativo 1 (MA1), Modelo Alternativo 2 (MA2) e Modelo Alternativo 3 (MA3), após ajuste pelos índices de modificação.

\begin{tabular}{|c|c|c|c|c|}
\hline \multirow{2}{*}{ Fatores } & \multicolumn{4}{|c|}{ Modelo (fatores) } \\
\hline & MB (7) & MA1 (5) & MA2 (6) & MA3 (3) \\
\hline $\mathrm{F} 1$ & $\begin{array}{l}\text { Gestão das Relações } \\
\text { Interpessoais }\end{array}$ & $\begin{array}{l}\text { Gestão das Relações } \\
\text { Interpessoais }\end{array}$ & Segurança & Poder \\
\hline $\mathrm{F} 2$ & $\begin{array}{l}\text { Comprometimento } \\
\text { Organizacional }\end{array}$ & $\begin{array}{l}\text { Comprometimento } \\
\text { Organizacional }\end{array}$ & Estratégia & Realização \\
\hline F3 & Trabalho em Equipe & Trabalho em Equipe & Remuneração & Afiliação \\
\hline F4 & Carga de Trabalho & Carga de Trabalho & Liderança & --- \\
\hline F5 & $\begin{array}{l}\text { Expectativa de } \\
\text { Desempenho }\end{array}$ & $\begin{array}{l}\text { Expectativa de } \\
\text { Desempenho }\end{array}$ & Relação com a Comunidade & --- \\
\hline F6 & Suporte Material & --- & $\begin{array}{l}\text { Desenvolvimento Profis- } \\
\text { sional }\end{array}$ & --- \\
\hline F7 & Valorização do Trabalho & --- & --- & --- \\
\hline
\end{tabular}

É interessante notar a pouca diferença entre os valores de um mesmo indicador na Tabela 1. Exceção aos valores de AIC. Por mais que os dados sejam oriundos de uma mesma base de dados, a maneira como foram combinados foi diferente. Se por um lado não se pode afirmar qual o melhor ou o pior modelo por meio desses indicadores, pode-se afirmar que eles compartilham algum grau de similitude a despeito das diferenças estruturais.

Esses resultados trazem dois aspectos relevantes. Por um lado, enfatiza a importância do uso de diferentes indicadores de ajuste para avaliar o modelo. De outro, a possibilidade de modelos diferentes medirem o mesmo construto por meio de aspectos distintos desse construto.

Para comparar os modelos e identificar qual o melhor, os resultados de ECVI (Expected Cross-Validation Index) da Tabela 3 serão utilizados. Como dito anteriormente, os modelos não são diretamente comparáveis. Porém, ECVI permite um "ordenamento" dos modelos. Os melhores modelos são os que apresentam o menor ECVI. A técnica de bootstrapping indica que os quatro modelos concorrentes são significativamente diferentes. Também aponta MA3 como o melhor. A técnica de cross-validation mostrou que MA2 e MB não apresentam diferenças significativas, mas concorda ao apontar MA3 como o modelo de melhor ajuste. Esses resultados indicam que a técnica de bootstrapping é um pouco menos sensível do que a de cross-validation para diferenciar modelos. Dada a simplicidade de aplicação do bootstrapping, recomenda-se o seu uso para identificar o melhor modelo de um grupo de modelos, mas não para discernir vários modelos concorrentes entre si. Para este último, sugere-se a cross-validation.

A hipótese de que o modelo com o maior número de fatores implica em melhor ajuste não se confirmou. $\mathrm{O}$ com mais fatores foi o de pior ajuste. Os resultados até aqui obtidos sugerem que modelos com mais fatores explicam mais variância, porém o número maior de fatores e inter-relações implica em maior dificuldade de ajuste. Quanto ao suporte teórico, se por um lado a teoria ajuda a desenvolver o modelo, por outro, não garante que o modelo terá um bom ajuste. $\mathrm{O}$ ajuste se mostrou mais uma questão de ordem numérica, conforme sugere o efeito dos índices de modificação. Há ainda o agravante de que modelos com mais fatores tendem a depender menos desses índices para o seu ajuste.

A Tabela 4 mostra que a fidedignidade dos diferentes fatores é entre razoável e boa. Isso permite afirmar que as estruturas fatoriais propostas são suficientemente estáveis como instrumentos psicométricos.

A despeito do fato de MA3 ter o melhor ajuste, ainda assim não se pode ignorar o fato de que todos os modelos apresentaram ajustes razoáveis. Se se admite que os modelos meçam diferentes aspectos do mesmo construto, o uso combinado deles ajudará a melhor medir esse construto. Aqui cabe a ideia de dimensões específica-geral e descritiva-avaliativa apresentada por Patterson et al. (2004). Como já mencionado, a escolha de um modelo não dependerá apenas do seu grau de ajuste, mas também da sua aplicação.

\section{Aplicação dos modelos}

Ao se pensar sobre a aplicação de um modelo, a questão de "qual o melhor modelo para a pesquisa?" passa a ser "qual a melhor pesquisa para o modelo?". Essa forma diferente de indagar está relacionada não só com a complexidade de um construto multifatorial, mas com a própria limitação dos instrumentos em medir cada um dos fatores.

Retomando a ideia de Patterson et al. (2004), pode-se categorizar os problemas de pesquisa em quatro grupos: 1) pesquisas que procuram respostas a aspectos específicos do problema e com orientação descritiva (específica-descritiva); 2) pesquisas que procuram respostas específicas, porém de cunho avaliativo, querendo estabelecer uma escala de ordem 
(específica-avaliativa); 3) pesquisas que procuram analisar aspectos gerais do problema e com orientação descritiva (geral-descritiva); e, finalmente, 4) pesquisas em aspectos gerais, mas que procuram estabelecer uma escala de ordem (geral-avaliativa). A partir dessas categorias de pesquisa, é possível escolher o modelo mais apropriado.

Para pesquisas de dimensão avaliativa, os levantamentos são realizados em períodos regulares (medidas repetidas). Dependendo do tamanho da organização, o custo e o tempo envolvidos no levantamento são consideráveis. Instrumentos com menos itens implicam em aplicações mais expeditas e menos onerosas para coleta, tabulação e análise. Logo, recomendam-se modelos com menos fatores e itens.

Para pesquisas de dimensão descritiva, não existe a necessidade de regularidade, mas de detalhamento do fenômeno para a sua compreensão. Assim, modelos com mais fatores e que meçam variáveis mais desagregadas são mais recomendados.

Para pesquisas de dimensão específica, normalmente o estudo visa estabelecer ações pontuais de um gerente ou supervisor dentro da organização. A melhor abordagem é um modelo de mensuração baseado em uma teoria específica para o objeto de estudo escolhido (ênfase no indivíduo, ou na organização, ou na relação entre indivíduo e organização).

Para estudos de ordem geral, a preocupação do pesquisador é de buscar subsídios para compreender a articulação entre as variáveis endógenas e exógenas do problema e mesmo em diferentes níveis. Assim, modelos que incluam mais fatores e que meçam variáveis mais agregadas são os mais indicados. No presente trabalho, talvez até caiba a decisão pela mensuração do construto Cultura Organizacional, como definido por Schneider et al. (2002), ao invés de CLO.

\section{Ressalvas desta pesquisa}

Os modelos alternativos (MA1, MA2 e MA3) utilizados foram desenvolvidos a partir dos mesmos itens desenhados para MB. No caso de MA2 e MA3, isso implica em problemas conceituais que não tornariam esses modelos perfeitamente representativos das respectivas teorias. Era esperado que esses modelos se ajustassem pior que o $\mathrm{MB}$, o que não aconteceu.

Outra ressalva diz respeito ao número de itens em cada modelo. Após as modificações realizadas em cada modelo, MA1, MA2 e MB apresentaram um número superior a 30 itens. Apenas MA3 apresentou um número próximo a 20 itens. Assim, a vantagem de utilizar um modelo com poucos fatores a fim de permitir a construção de instrumentos menores, mais expeditos e de menor custo é verdade apenas para MA3. Um modelo também com 22 itens, mas distribuídos em quatro fatores, foi obtido por Bamel, Rangnekar, Stokes e Rastogi (2013).

\section{Conclusões}

A hipótese de que a melhor medida de CLO seria obtida com o modelo de mensuração com maior número de fatores não se confirmou. Os resultados indicam que os quatro modelos concorrentes comportam-se de maneira similar. Todos apresentaram um ajuste razoável. As técnicas de bootstrapping e cross-validation apresentaram resultados um pouco diferentes, mas apontaram MA3 como o de melhor ajuste. Para se identificar o melhor modelo, sugere-se o uso do bootstrapping pela simplicidade de aplicação. Contudo, para discernir entre modelos de mensuração, a técnica de cross-validation mostrou-se mais sensível.

Em situações onde os modelos concorrentes apresentam ajustes semelhantes, a escolha do modelo deve ser calcada na finalidade da pesquisa. A finalidade de uma pesquisa pode ser determinada em duas dimensões: 1) específica-geral; e 2) descritiva-avaliativa.

Em termos de teoria, as duas teorias utilizadas (teoria da motivação e teoria do campo vital) mostraram-se proxies válidos da medida de CLO.

Cabem algumas considerações para cada modelo:

- MB não é um bom modelo em termos de ajuste, mas é o que proporciona maior percentual de explicação da variância. Esse modelo oferece mais subsídios aos gestores sobre as fontes de gargalo do desempenho da organização.

- MA2 não se mostrou significativamente diferente de $\mathrm{MB}$, em termos de ajuste. Porém, a fidedignidade dos seus fatores é melhor do que a de MB.

- MA1 tem um número maior de itens do que MA3 e um número inferior de fatores do que MA2 e MB. Isso o classifica como um modelo intermediário, sem vantagens significativas em relação aos demais modelos de mensuração. Dada a falta de suporte teórico, não se recomenda o seu uso.

- MA3, mais simples, com questionário mais curto, é uma ferramenta mais interessante quando o número de respondentes é grande e o objetivo do levantamento é de monitorar o estado da organização.

A partir dos resultados desta pesquisa, sugere-se que futuros estudos sejam feitos a fim de desenvolver novos instrumentos psicométricos específicos para as teorias de Lewin ou de McClelland.

\section{Referências}

Akaike, H. (1987). Factor analysis and AIC. Psychometrika, 52(3), 317-332.

Ancarani, A., Di Marco, C., \& Giammanco, M. D. (2011). Patient satisfaction, managers' climate orientation and organizational climate. International Journal of Operations \& Production Management, 31(3), 224-250. doi:10.1108/01443571111111900 
Arabaci, I. B. (2010). Academic and administration personnel's perceptions of organizational climate (Sample of Educational Faculty of Firat University). Procedia Social and Behavioral Sciences, 2, 4445-4450.

Arbuckle, J. L. (2009). Amos 18 User's Guide. Crawfordville, Florida: Amos Development Corporation.

Argyris, C. (1958). Some problems in conceptualizing organizational climate: A case study of a bank. Administrative Science Quarterly, 2(4), 501-520.

Bamel, U. K., Rangneskar, S., Stokes, P., \& Rastogi, R. (2013). Organizational climate and managerial effectiveness: An Indian perspective. International Journal of Organization Analysis, 21(2), 198-218. doi:10.1108/IJOA-09-2011-0514

Bhutto, N. A., Laghari, M. K., \& Butt, F. (2012). A comparative study of organizational climate and job satisfaction in public, private and foreign banks. Asian Social Science, 8(4), 259-267. doi:10.5539/ass.v8n4p259

Brown, T. A. (2006). Confirmatory factor analysis for applied research. New York: The Guilford Press.

Byrne, B. M. (2001). Structural equation modeling with Amos: Basic concepts, applications, and programming. Mahwah, New Jersey: Lawrence Erlbaum Associates.

Carr, J. A., Schmidt, A. M., Ford, J. K., \& DeShon, R. P. (2003). Climate perceptions matter: A meta-analytic path analysis relating molar climate, cognitive and affective states, and individual level work outcomes. Journal of Applied Psychology, 88(4), 605-619.

Chan, D. (1998). Functional relations among constructs in the same content domain at different levels of analysis: A typology of composition models. Journal of Applied Psychology, 83(2), 234-246.

Gavin, J. F. (1975). Organizational Climate as a function of personal and organizational variables. Journal of Applied Psychology, 60(1), 135-139.

Gomes, F. R. (2002). Clima organizacional: um estudo em uma empresa de telecomunicações. Revista de Administração de Empresas, 42(2), 95-103.

Guion, R. M. (1973). A note on organizational climate. Organizational Behavior and Human Performance, 9(1), 120-125. doi:10.1016/0030-5073(73)90041-X

Hélie, S. (2006). An introduction to model selection: Tools and algorithms. Tutorials in Quantitative Methods for Psychology, 2(1), 1-10.

James, L. R., \& Jones, A. P. (1974). Organizational climate: A review of theory and research. Psychological Bulletin, 81(12), 1096-1112.

James, L. R., Choi, C. C., Ko, C. E., McNeil, P. K., Minton, M. K., Wright, M. A., \& Kim, K. (2008). Organizational and psychological climate: A review of theory and research. European Journal of Work and Organizational Psychology, 17(1), 5-32.

Kolb, D. A. (1978). Organizational psychology: An experimental approach. New Jersey: Prentice Hall.

Kolb, D. A., Rubin, I. M., \& McIntyre, J. M. (1986). Psicologia organizacional: uma abordagem vivencial. São Paulo, SP: Atlas.
Laros, J. A., \& Puente-Palacios, K. E. (2004). Validação cruzada de uma escala de clima organizacional. Estudos de Psicologia, 9(1), 113-119.

Lewin, K. (1939). Field Theory and experiment in social psychology: Concept and methods. American Journal of Sociology, 44(6), 868-896.

Lewin, K., Lippitt, R., \& White, R. (1939). Patterns of aggressive behaviors in experimentally created social climates. Journal of Social Psychology, 10, 271-299.

Maximiano, A. C. A. (2000). Introdução à administração (5 ed.). São Paulo, SP: Atlas.

McClelland, D. (1955). Studies in motivation. New York: Appleton.

McClelland, D. (1972). A sociedade competitiva: realização e progresso social. Rio de Janeiro: Expansão e Cultura.

Menezes, I. G., Sampaio, L. R., Gomes, A. C. P., Teixeira, F. S., \& Santos, P. de S. (2009). Escala de clima organizacional para organizações de saúde: desenvolvimento e estrutura fatorial. Estudos de Psicologia, 26(3), 305-316.

Moran, E. T. \& Volkwein, J. F. (1992). The cultural approach to the formation of organizational climate. Human Relations, 45(1), 19-48.

Myers, R. (1990). Classical and modern regression with applications. Boston, Massachusetts: Duxbury.

Nakata, L. E., Veloso, E. F. R., Fischer, A. L., \& Dutra, J. S. (2009). Uso de pesquisas de clima organizacional no Brasil. Gestão Contemporânea, 6(6), 45-68.

Ostroff, C. (1993). The effects of climate and personal influences on individual behavior and attitudes in organizations. Organizational Behavior and Human Decision Processes, 56, 56-90.

Patterson, M., Warr, P., \& West, M. (2004). Organizational climate and company productivity: The role of employee affect and employee level. Journal of Occupational and Organizational Psychology, 77, 193-216.

Puente-Palacios, K. E. (2002). Abordagens teóricas e dimensões empíricas do conceito de clima organizacional. Revista de Administração, 37(3), 96-104.

Sá Leitão, J., Guimarães, T., \& Azevedo-Rosal, M. de (1998). Metodologia de diagnóstico de clima organizacional em ambiente de inovação tecnológica. In Associação Nacional dos Programas de Pós-Graduação em Administração (Ed.), Encontro Anual da Associação Nacional dos Programas de Pós-Graduação em Administração, 22. Foz de Iguaçu, PR: ANPAD. Disponível em: http://www.anpad.org.br/evento. php? acao $=$ trabalho\&cod_edicao_subsecao $=53 \&$ cod_evento edicao $=2 \&$ cod_edicao_trabalho $=3708$

Sbragia, R. (1983). Um estudo empírico sobre o clima organizacional em instituições de pesquisa. Revista de Administração da USP, 18(2), 30-39.

Schneider, B. (1985). Organizational behavior. Annual Review of Psychology, 36, 573-611.

Schneider, B., Salvaggio, A. N., \& Subirats, M. (2002). Climate strength: A new direction for climate research. Journal of Applied Psychology, 87(2), 220-229. 
Schulte, M., Ostroff, C., Smulyian, S., \& Kinicki, A. (2009). Organizational climate configurations: Relationships to collective attitudes, customer satisfaction, and financial performance. Journal of Applied Psychology, 94(3), 618-634. doi: 10.1037/a0014365

Souza, E. L. P. de. (1982). Clima e motivação em uma empresa estatal. Revista de Administração de Empresas, 22(1), 14-18.

Stone, P. W., \& Gershon, R. R. M. (2006). Nurse work environments and occupational safety in Intensive Care Units. Policy, Politics \& Nursing Practice, 7(4), 240-247.

Tabachnick, B. G., \& Fidell, L. S. (2001). Using multivariate statistics (4th ed.). Boston, Massachusetts: Allyn \& Bacon.

Thompson, B. (2004). Exploratory and confirmatory factor analysis. Washington, DC: American Psychological Association.
Ullman, J. B. (2006). Structural Equation Modeling: Reviewing the basics and moving forward. Journal of Personality Assessment, 87(1), 35-50.

Wood, R., \& Bandura, A. (1989). Social cognitive theory of organizational management. The Academy of Management Review, 10(3), 361-384.

Recebido em 23.06.2012

Primeira decisão editorial em 30.08.2013

Versão final em 28.10.2013

Aceito em 17.01.2014 